Published in final edited form as:

Clin Gastroenterol Hepatol. 2015 November ; 13(12): 2166-2172. doi:10.1016/j.cgh.2015.07.015.

\title{
Hepatitis C Virus Treatment: Is It Possible To Cure All Hepatitis C Virus Patients?
}

\author{
Andrew J. Muir ${ }^{*}, \ddagger$ and Susanna Naggie ${ }^{*}, \S$ \\ *Duke Clinical Research Institute, Duke University School of Medicine, Durham, North Carolina \\ ‡Division of Gastroenterology, Department of Medicine, Duke University School of Medicine, \\ Durham, North Carolina \\ §Division of Infectious Diseases, Department of Medicine, Duke University School of Medicine, \\ Durham, North Carolina
}

\section{Abstract}

The recent advances in hepatitis $\mathrm{C}$ virus $(\mathrm{HCV})$ therapeutics have brought combinations of direct acting antiviral medications that offer interferon-free, well-tolerated regimens with sustained virologic response rates greater than $90 \%$ in clinical trials for many patient groups. The successes have prompted discussions regarding cure for all patients. These regimens have already demonstrated the ability to cure previously challenging patient groups, including human immunodeficiency virus-HCV coinfection, decompensated cirrhosis, and post-liver transplantation. Limitations exist in the current portfolio of agents, with suboptimal outcomes for genotype 3 and limited data in genotypes 5 and 6 . More data are urgently needed in patients with chronic kidney disease and in children. With ongoing developments, highly effective regimens for all these patient groups are within reach. To deliver HCV treatment throughout the world and particularly in low-and middle-income countries, regimens need to be affordable but also pangenotypic, well-tolerated, and delivered once daily for 4-8 weeks. With such a regimen, cure for all patients would then hinge on the ability to identify patients with $\mathrm{HCV}$ infection and deliver treatment within their communities. This review will discuss the strategies that will be necessary to realize this opportunity to cure all persons with $\mathrm{HCV}$ infection.

\section{Keywords}

Hepatitis C; Antiviral Agents; Diagnosis; Disease Management

Is it possible to cure all hepatitis $\mathrm{C}$ virus (HCV) patients? Yes, but we have not yet realized the true potential of direct acting antivirals (DAAs) for HCV infection. The recent advance

Reprint requests: Address requests for reprints to: Andrew J. Muir, MD, MHS, DUMC Box 3913, Durham, North Carolina 27710. ; Email: andrew.muir@ duke.edu; fax: (919) 684-8264

Conflicts of interest

The authors disclose the following: Dr Muir has received research funding from AbbVie, Bristol-Myers Squibb, Gilead Sciences, Inc, Janssen Therapeutics, Merck \& Co, Inc, Roche, and Vertex Pharmaceuticals and has served as a scientific advisor to AbbVie, BristolMyers Squibb, Gilead Sciences, Inc, Janssen Therapeutics, Merck \& Co, Inc, and Theravance. Dr Naggie has received research funding from AbbVie, Bristol-Myers Squibb, Gilead Sciences, Inc, Janssen Therapeutics, and Vertex Pharmaceuticals, Inc and has served as a scientific advisor to AbbVie, Bristol-Myers Squibb, Merck \& Co Inc, and Gilead Sciences, Inc. 
in HCV therapeutics is one of the major accomplishments of modern medicine. After more than 20 years of interferon-based regimens, which offered moderate efficacy with significant morbidity and had a safety profile that limited its use across many populations, a number of DAA medications have been approved and are now available. These agents in combination offer interferon-free, well-tolerated regimens with sustained virologic response (SVR) rates greater than $90 \%$ in clinical trials. ${ }^{1 \_3}$ Therefore, we have the tools to cure patients who engage in care, but therein lies the rub. As we hear the calls for global eradication, there remain many challenges in the pursuit of "cure for all."

\section{Antiviral Therapy}

Broad scale treatment of $\mathrm{HCV}$ has only become a reality with the development of welltolerated, all oral DAA combination therapies. The safety profile of interferon-based regimens created a tremendous barrier to the treatment of many patients and impacted the enthusiasm of patients and providers alike. With less than 50\% SVR effectiveness in the most common $\mathrm{HCV}$ genotype worldwide, it was difficult for many providers to endorse screening programs because they knew that the patient may receive a diagnosis for a disease that could not be adequately treated. DAA combination therapies now offer SVR rates greater than $90 \%$ for treatment-naive and experienced patients with genotypes 1 through 4 . The dramatic improvement in efficacy along with a similarly monumental advance in safety has created the opportunity for treatment for all. One especially positive sign of progress in the field has been the inclusion of previously excluded special populations in development programs including patients with human immunodeficiency virus (HIV)-HCV coinfection, decompensated cirrhosis, and liver transplantation. ${ }^{4}{ }^{7}$ For HIV/HCV patients in particular, SVR rates are similar to HCV monoinfected patients, resulting in a national dialogue as to whether HIV coinfection should maintain the label of a special population.

Despite these advances, current regimens are imperfect, and albeit easier than before, treatment remains complex. Table 1 summarizes the successes and limitations of current treatments. Although we can cure the majority of patients with genotypes 1, 2, and 4 in 12 weeks, many genotype 1 patients and all genotype 3 patients require 24 weeks of treatment. ${ }^{8}$ Viewed by many as the new difficult-to-treat genotype, SVR rates for genotype 3 patients with cirrhosis have not reached the $90 \%$ SVR threshold observed in other patient groups. ${ }^{9}$ We have limited data in patients with genotype 5 and 6 infection and few safe options for patients with chronic kidney disease. No DAA agents have been approved for patients with end-stage renal disease, and the continued requirement of ribavirin for most regimens in various patient subgroups is especially challenging for patients with chronic kidney disease because of the risk of severe anemia. In fact, we have yet to establish the appropriate dose of ribavirin when used as a component of DAA combination therapies. There remains a laundry list of patients who have been excluded from clinical trials that leave clinicians struggling with the best and safest approach, including but not limited to HIV patients on complex antiretroviral regimens, immunocompromised hosts, patients with hepatitis B virus coinfection, children, and the elderly, who represent an increasingly recognized and identified subgroup of patients living with HCV. 
In addition to those historic "special populations", there is a new, steadily growing population for whom we have little to offer. Already, as we collect more patients treated and exposed to DAA regimens without success, we are learning that viral resistance has a role to play in retreatment success. Is there a price to pay for DAA treatment failure, other than the cost of the failed regimen itself? Early reports of baseline resistance associated variants (RAVs) suggest that the answer may be yes at a minimum in some of our more difficult-totreat patient subgroups. Sofosbuvir, a NS5B nucleotide analogue, has an exceptional genetic barrier to resistance. The signature RAV with sofosbuvir is the S282T variant, which has never been detected naturally at baseline because of the extremely poor fitness of the variant and is rarely identified at the time of sofosbuvir failure. ${ }^{10}$ Patients failing sofosbuvir onlycontaining regimens have been successfully re-treated with a sofosbuvir-containing DAA combination regimen. ${ }^{11}$ Baseline and treatment emergent variants (TEVs) have been observed with the NS3/4A protease inhibitors and the NS5A inhibitors. ${ }^{12}$ The degree of the impact of RAVs on treatment outcomes with these potent antiviral regimens remains unknown, but the development of resistance at the time of treatment failure is almost universal. NS5A RAVs maintain remarkable fitness and are likely to be more problematic in the setting of retreatment. In a pooled resistance analysis of the ledipasvir (NS5A inhibitor)/ sofosbuvir phase 3 programs, although only $16 \%$ of patients overall had NS5A RAVs at baseline, significantly more patients (43\%) suffering virologic failure harbored these RAVs at baseline. ${ }^{13}$ There are now several patient subgroups where baseline NS5A RAVs may lower SVR including genotype 3 patients with cirrhosis treated with daclatasvir (NS5A inhibitor) and sofosbuvir and treatment-experienced patients receiving ledipasvir and sofosbuvir. $^{14}$

Patients harboring TEVs are likely to become a rapidly expanding patient subgroup with significant need. Although SVR of 95\%-99\% in clinic trials suggests this population should be a minor subgroup, it is unlikely that clinical practice will achieve such perfection. We can expect to see the proportion of treatment failures grow as the treatments move outside of clinical trials and into clinical practice. Poor adherence and the presence of RAVs have been reported most often as reasons for treatment failure in the clinical trials with these regimens. Adverse events have not been a common reason for discontinuation and treatment failure. Registries will be important to understand outcomes and reasons for failure as treatment expands outside experienced centers. Viral resistance testing is available commercially but is not currently recommended in routine clinical practice and yet may become important in the identification of the most effective regimens for patients with prior DAA treatment failure. Efforts are also underway to evaluate potent regimens with multiple agents for longer durations for this group of patients. The rapid pace of developments in DAA regimens has led to confidence that in time, all patients will have a regimen that offers cure of $\mathrm{HCV}$.

If our goal is to cure everyone of $\mathrm{HCV}$ infection as part of a campaign to eradicate $\mathrm{HCV}$, we will need a different arsenal than that we have now. Even if we just focus on the genotypes and patient groups with excellent SVR rates to current treatment, there are obstacles that will impact broad scale-up of treatment across the globe. Current regimens vary according to the genotypes and subtypes, and some regimens still require ribavirin, leading to anemia with requisite monitoring. Treatment durations currently range from 8 to 24 weeks depending on absence or presence of cirrhosis, treatment history, and baseline HCV RNA. This results in a 
complex treatment paradigm that will remain a barrier to treatment uptake in nonsubspecialty clinics and low- and middle-income countries, which are the very places we will need the greatest presence if we are going to achieve this goal of cure for all.

One strategy of current research programs is to develop pan-genotypic regimens of short duration across all patient subgroups (Figure 1). Removing the requirement for genotype testing reduces the cost of the diagnostic evaluation and the required resources that may not be available in many areas of the world. A pan-genotypic regimen also decreases the complexity of the treatment paradigm, increasing the likelihood that clinicians other than $\mathrm{HCV}$ experts can get involved in care. The nucleotide inhibitor sofosbuvir is pan-genotypic and an attractive component of such a regimen. ${ }^{1}$ The NS5A inhibitor daclatasvir is available in Europe, with clinical data available in genotypes 1-4 with 12 weeks in treatment-naive patients and replicon data to support the use in genotypes 5 and $6 .{ }^{15}$ The regimen of sofosbuvir and the NS5A inhibitor GS-5816 is also in development as a pan-genotypic regimen. ${ }^{16}$ In addition, second-generation NS3-4A protease inhibitors (including MK-5172 and GS-9857) offer pan-genotypic activity and create the potential for highly potent, triple pan-genotypic DAA combination therapies that probably provide the greatest hope for a standardized approach to the treatment of $\mathrm{HCV}$ across the globe. ${ }^{17}$ As these regimens are developed, SVR rates must remain in the current range of 95\%-99\% if the goal is HCV eradication. If regimens with lower efficacy rates are endorsed to promote widespread use, we will see more treatment failure and with it more resistant variants. Although shorter duration will be important to achieve broad treatment of HCV throughout the world, this cannot come with a cost in efficacy.

Thus, the optimist would theorize that continued progress in drug development will soon lead to an ideal regimen that is pan-genotypic, once daily, and well-tolerated and cures all patient populations in 4-8 weeks. Our ability to cure all patients then hinges on economics and the operational components of treatment. The costs of HCV treatment have been the subject of much scrutiny in high-income countries with access to these medications. A recent cost-effectiveness analysis demonstrated that treatment of all eligible HCV-infected Americans with sofosbuvir-based regimens was cost-effective compared with the previous standard of care. This treatment program would cost an additional $\$ 65$ billion in the next 5 years, with the resulting cost offsets of $\$ 16$ billion. ${ }^{18}$ Although market forces are expected to influence these issues in the high-income countries of the world, the costs of these medicines are prohibitive to the low- and middle-income countries. Similar issues were faced for antiviral medications for HIV, and only through the development of generics did broad treatment occur. Gilead, the manufacturer of sofosbuvir, issued voluntary licenses to Indian generic companies to produce and market sofosbuvir in 91 countries. ${ }^{19}$ Similar steps will be needed for all DAAs including those in combination before we can offer universal access to HCV cure.

\section{Hepatitis C Virus Screening and Diagnosis}

The advances in antiviral therapy with highly effective and well-tolerated oral regimens are critical to the efforts to pursue cure for all with HCV. For the medications to achieve their potential, we need more effective programs to identify persons living with HCV infection. 
The availability of effective medications has energized efforts to combat $\mathrm{HCV}$ at the population level. Previous screening initiatives were negatively impacted by the harsh side effects and meager response rates of interferon-based regimens. It is estimated that less than half of Americans are aware of their HCV infection, and there are countries where the prevalence of infection is simply unknown. ${ }^{20}$ Our previous approach to HCV diagnosis and treatment improved the health of very few persons, with an estimated 5\%-6\% of the American HCV population cured of HCV (Figure 2). Thus, to maximize the impact of monumental advances in $\mathrm{HCV}$ therapeutics, the identification of more patients with $\mathrm{HCV}$ is desperately needed.

Because of the asymptomatic nature of chronic infection, screening programs are required to uncover the burden of this "silent infection." In the United States, screening of persons at risk for $\mathrm{HCV}$ infection has been recommended since $1998 .^{21}$ These initial recommendations were based on risk factors for $\mathrm{HCV}$ infection including injection drug use and transfusion before 1992. It has been generally accepted that the initial American HCV screening programs failed. The stigma associated with injection drug use was strongly implicated as a driver of this failure because of the lack of willingness for patients to report this prior risk exposure to their providers. As a result, the Centers for Disease Control and Prevention recommended screening of the baby boomer cohort born between 1945 and 1965 because this group carries approximately $75 \%$ of $\mathrm{HCV}$ infections in the United States. ${ }^{22}$ Ongoing initiatives will determine whether this strategy is successful. The lack of a national surveillance program for chronic $\mathrm{HCV}$ infection limits our ability to assess the impact of these initiatives.

Successful screening initiatives will require that primary care providers understand HCV epidemiology and the diagnostic evaluation and will command a basic understanding of $\mathrm{HCV}$ treatment and its value. Previous studies have suggested that knowledge of HCV among primary care providers is limited. ${ }^{23}$ Because of the stigma of HCV infection and the role of nurses and other health care team members in screening programs, broad training will be necessary. ${ }^{24}$ Screening initiatives that are burdensome on the clinical practice or health care system will be limited in their success because of the competing demands of busy clinicians. Electronic reminders have been effectively used in HCV screening programs but are not widely used. ${ }^{25}$ Epic, the largest electronic health record vendor in the United States, has loaded content to provide electronic reminders and population management tools to support HCV testing in the 1945-1965 birth cohort, but the individual hospitals or health systems need to activate the function for the potential to be realized. They have also developed educational content on the rationale for screening and a library to share the HCV decision support programs from other Epic centers. These programs are examples of the potential for population management within our health system through effective use of technology.

Most current screening programs target patients already engaged in health care. However, the epidemiology would suggest that many patients with HCV infection may not be currently in care and attending health care centers. The National Health and Nutrition Examination Survey has provided our best understanding of the HCV population in the United States. Of the 3-5 million Americans estimated to have HCV infection, important 
defining characteristics are that $63 \%$ are male, $23 \%$ are black, and $30 \%$ have a family income below the federal poverty level. ${ }^{26}$ Data from the prison population in the United States suggest chronic infection rates of $12 \%-35 \%{ }^{27}$ Efforts will be needed to identify strategies for screening in other settings and particularly in the community to engage these high-risk populations. Because many within these populations seek only emergent or urgent care, a novel approach to screening has proven successful at the University of Alabama Birmingham, where patients presenting to the emergency department were screened regardless of their presenting complaint. During the study period, $1529 \mathrm{HCV}$ antibody tests were performed, and $11.1 \%$ were positive. ${ }^{28}$ In Europe, the French health care system has conducted the most successful national program to increase awareness and identify more patients with HCV. Their program was launched in 1999 and included outreach initiatives to persons who inject drugs, immigrant communities from high prevalence countries, and prison populations. An analysis from 1994 to 2006 reported that the proportion of persons aware of HCV increased from $24 \%$ to $56 \% .^{29}$ Similar strategies will be important components to any national strategy.

For those groups and countries attempting to battle HCV in resource-limited settings, the current diagnostic tests are limiting. Although rapid tests amenable to field work are available for the HCV antibody, confirmation of HCV infection with HCV RNA is not available. ${ }^{30}$ Improvements in diagnostics will be necessary to bring effective screening and treatment programs to resource-limited regions of the world.

\section{Linkage to Care and Treatment Programs}

Once the diagnosis of HCV infection is made, that initial step must be followed by linkage to care and initiation of treatment when indicated. In the United States, HCV treatment is currently delivered by a relatively small number of clinicians trained in gastroenterology, hepatology, or infectious diseases who are based in academic centers and referral practices. As demonstrated by the Centers for Disease Control and Prevention analysis (Figure 1), patients are lost from our care continuum at each step toward HCV treatment. Only half of Americans with HCV have been made aware of the diagnosis, and then only $32 \%-38 \%$ have been referred for specialty care, with treatment delivered to $7 \%-11 \%{ }^{20}$ These statistics were clearly impacted by the presence of the interferon-based regimens, which were great deterrents for patients and providers to embark on antiviral treatment. However, other barriers exist for both patients and clinicians in their efforts to receive HCV care. Persons infected with HCV still suffer stigma related to HCV infection and may be reticent to move forward in care. Persons with HCV have increased prevalence of comorbid mental illness and substance abuse as well as lower education and income levels. ${ }^{31}$ Interventions to improve linkage to care have found that care coordination and patient navigator services are effective, but they cannot overcome barriers that include availability of specialty care and lack of health insurance coverage. ${ }^{32,33}$ Treatment centers are not available in all geographic regions of the United States, and patients may be unable or unwilling to travel great distances. Project ECHO overcame many of these barriers by offering care in the rural communities of the patients. Primary care providers effectively offered HCV treatment with support from specialists in the academic center. ${ }^{34}$ More efforts are needed to offer treatment in the communities of persons living with HCV infection. Epidemiologic data would support 
that populations of $\mathrm{HCV}$ patients are in federally qualified health centers, methadone clinics, substance abuse programs, mental health centers, and prisons. ${ }^{26}$ A pan-genotypic welltolerated regimen could be offered in these settings and may be the only way to successfully engage many of these patients living with $\mathrm{HCV}$ infection. These economic and geographic barriers are only more extreme in the low- and middle-income countries, and HCV treatment needs to be introduced into the existing public health infrastructure.

\section{Prevention}

Previous efforts to eradicate smallpox and gain control of other infections like polio have relied on a vaccine strategy to develop herd immunity. ${ }^{35}$ With no HCV vaccine expected in the near future, current efforts are evaluating whether antiviral treatment regimens can play a similar role in impacting the prevalence in a population. Time will tell whether this is an effective strategy, but it is clear that antiviral therapy will be unable to control HCV without aggressive efforts to prevent new infections. The strategies for prevention will also vary according to the country and transmission routes. The incidence of acute HCV infection steadily declined in the United States from 1992 until 2005, and these decreases were believed to be related to screening of the blood supply and strategies to prevent transmission of HIV/acquired immunodeficiency syndrome. The plateau was followed by an increase of $45 \%$ in reported acute HCV infections in 2011, with 15 states responsible for the surge. ${ }^{36}$ Investigations of specific outbreaks have linked these increases to injection drug use among teenagers and young adults. ${ }^{37,38}$ Needle exchange programs and substance abuse treatment programs are not widely available in the United States and will be critical to gain control of $\mathrm{HCV}$ in this population. In other parts of the world, many HCV infections occur in health care settings when receiving injections or blood products. Egypt provides the best historical example when unsafe needle practices in their Schistosoma mansoni control campaign led to widespread transmission of $\mathrm{HCV}^{39}$ Unsafe injection practices remain a major source of HCV transmission throughout the world. These exposures as well as blood product, organ, and tissue donations have been estimated to result in 2 million new infections per year worldwide. ${ }^{40}$ A 2011 World Health Organization report noted that 39 countries did not screen the blood supply for transfusion-transmissible infections including HIV, hepatitis B, hepatitis $\mathrm{C}$, and syphilis. In addition, $47 \%$ of blood donations in low-income countries were being tested in laboratories without quality assurance. ${ }^{41}$ These numbers are troubling, but a recent report did show progress. In a survey comparing practices from 2000 to 2004 with 2010 to 2011, the number of sub-Saharan African countries testing at least $95 \%$ of donations for HCV antibody increased from 13 of $38(34 \%)$ to 30 of $35(86 \%) .{ }^{41}$ Similar efforts around prevention will be crucial to any national program to eradicate $\mathrm{HCV}$.

\section{National Strategies}

Once these potent, short, and pan-genotypic regimens have been identified, strategies will be needed to guide the approach to treatment on a population level. When thinking about HCV throughout the world, the availability of care varies broadly. In countries such as Egypt with strong health care infrastructure and the political will to battle HCV, effective screening and treatment programs have been developed. ${ }^{42}$ Many countries in the world have not addressed HCV with a national strategy. In 2014, the World Health Organization released guidelines to 
provide the outline for national HCV programs for prevention, diagnosis, and treatment. ${ }^{43}$ Leadership and commitment at the highest levels of government will be required for these programs to succeed. Only those countries making this commitment to a national program can realistically hope to address $\mathrm{HCV}$ and participate in the dialogue of eradication. Within these national programs, regional and local strategies will need to consider a number of issues and perhaps prioritize patient groups according to local epidemiology, culture, and infrastructure. Persons who inject drugs have high prevalence of HCV infection, and screening and treatment in this population would identify a large number of patients efficiently and provide an opportunity to lower prevalence and possibly transmission. Patients with HIV-HCV coinfection should be prioritized because of their more aggressive natural history and increase in liver-related mortality. ${ }^{44}$ In countries with established infrastructure to care for patients with HIV, the addition of HCV care will be more easily accomplished than in settings where the infrastructure must be created. To achieve widespread HCV care in all parts of the world, clinicians from a variety of backgrounds will need to be engaged and trained. Training, diagnostic, and treatment algorithms and support from local and regional experts will be necessary to achieve successful treatment outcomes.

\section{The Strategy in Durham}

Our clinics at Duke have been offering these interferon-free, well-tolerated oral regimens for several years through clinical trials. Therefore, we knew the potential of these regimens and the opportunity to impact HCV at the population level. In 2012, Dr Arlene Sena-Soberano in her role as Medical Director of the Durham County Health Department convened a working group to develop a local strategy for disadvantaged persons with HCV in Durham. The clearly stated goal was to cure every person in Durham with HCV infection. Dr SenaSoberano received a grant from Centers for Disease Control and Prevention to screen 2000 residents of Durham for $\mathrm{HCV}$, and screening initiatives started at the federally qualified health center, the county health department, sexually transmitted disease clinic, the homeless shelter, substance abuse treatment programs, and the Durham County jail. Patients diagnosed with $\mathrm{HCV}$ received assistance from a bridge counselor to enroll them in primary care if needed and obtain specialty consultation for HCV from physicians at Duke or the University of North Carolina Chapel Hill. This program has been effective in identifying patients and enrolling them in care, but too few patients have received treatment. Our biggest barrier has been the ability to afford the medications. North Carolina did not expand Medicaid with Obamacare, and many of these patients have no health coverage. Our strategy has been to take each patient one by one and try to offer them treatment through patient assistance programs or through clinical trials. Despite our efforts, we have also found that some patients have been unwilling or unable to attend clinics at the academic centers. It has become clear that we must facilitate treatment in the Durham community and away from the academic center if we expect to achieve our goal to cure all. Therefore, we have started to partner with primary care providers in Durham to offer HCV treatment. We have conducted training sessions to prepare these providers, and we expect treatment to begin in these centers soon. 


\title{
Conclusions
}

HCV antiviral medications already cure more than $90 \%$ of the HCV population including patients with HIV-HCV, decompensated cirrhosis, and post-transplant. Development programs are currently working toward a once daily, pan-genotypic regimen that cures $95 \%$ or more. These pan-genotypic regimens would be the basis for diagnostic and treatment algorithms that could be safely offered in a primary care clinic, methadone program, or prison in the United States or in any country of the world. As these regimens reach the clinics, the challenge will be to identify and treat patients with HCV. Screening programs have been limited to this point, and many patients do not have access or cannot afford these medications. The development of generic versions of DAAs and other efforts to bring down costs are needed. Leaders of governments throughout the world must commit for these programs to be successful, and local leaders will need to design programs that are culturally appropriate for the population at risk. We will be able to cure all HCV patients, but there remains much work to be done.

\author{
Abbreviations used in this paper \\ DAA \\ HCV \\ HIV \\ RAV \\ SVR \\ TEV \\ direct acting antiviral \\ hepatitis $\mathrm{C}$ virus \\ human immunodeficiency virus \\ resistance associated variant \\ sustained virologic response \\ treatment emergent variant
}

\section{References}

1. Lawitz E, Mangia A, Wyles D, et al. Sofosbuvir for previously untreated chronic hepatitis C infection. N Engl J Med. 2013; 368:1878-1887. [PubMed: 23607594]

2. Sulkowski MS, Gardiner DF, Rodriguez-Torres M, et al. Daclatasvir plus sofosbuvir for previously treated or untreated chronic HCV infection. N Engl J Med. 2014; 370:211-221. [PubMed: 24428467]

3. Poordad F, Hezode C, Trinh R, et al. ABT-450/r-ombitasvir and dasabuvir with ribavirin for hepatitis C with cirrhosis. N Engl J Med. 2014; 370:1973-1982. [PubMed: 24725237]

4. Kwo PY, Mantry PS, Coakley E, et al. An interferon-free antiviral regimen for HCV after liver transplantation. N Engl J Med. 2014; 371:2375-2382. [PubMed: 25386767]

5. Charlton M, Everson GT, Flamm SL, et al. Ledipasvir and sofosbuvir plus ribavirin for treatment of HCV infection in patients with advanced liver disease. Gastroenterology. 2015; 149:649-659. [PubMed: 25985734]

6. Sulkowski MS, Naggie S, Lalezari J, et al. Sofosbuvir and ribavirin for hepatitis $\mathrm{C}$ in patients with HIV coinfection. JAMA. 2014; 312:353-361. [PubMed: 25038354]

7. Sulkowski MS, Eron JJ, Wyles D, et al. Ombitasvir, paritaprevir co-dosed with ritonavir, dasabuvir, and ribavirin for hepatitis $\mathrm{C}$ in patients co-infected with HIV-1: a randomized trial. JAMA. 2015; 313:1223-1231. [PubMed: 25706092]

8. [Accessed June 1, 2015] Recommendations for testing, managing, and treating hepatitis C. 2015. Available at: http://hcvguidelines.org/ 
9. Zeuzem S, Dusheiko GM, Salupere R, et al. Sofosbuvir and ribavirin in HCV genotypes 2 and 3. N Engl J Med. 2014; 370:1993-2001. [PubMed: 24795201]

10. Svarovskaia ES, Dvory-Sobol H, Parkin N, et al. Infrequent development of resistance in genotype 1-6 hepatitis $C$ virus-infected subjects treated with sofosbuvir in phase 2 and 3 clinical trials. Clin Infect Dis. 2014; 59:1666-1674. [PubMed: 25266287]

11. Wyles D, Pockros P, Morelli G, et al. Ledipasvir-sofosbuvir plus ribavirin for patients with genotype 1 hepatitis $\mathrm{C}$ virus previously treated in clinical trials of sofosbuvir regimens. Hepatology. 2015; 61:1793-1797. [PubMed: 25846014]

12. McCormick AL, Moynihan L, Macartney MJ, et al. Baseline drug resistance mutations are detectable in HCV genes NS3 and NS5A but not NS5B in acute and chronic HIV-coinfected patients. Antivir Ther. 2015; 20:361-363. [PubMed: 25279548]

13. Sarrazin, C.; Dvory-Sobol, H.; Svarovskaia, E. Baseline and post-baseline resistance analyses of phase 2/3 studies of ledipasvir/sofosbuvir \pm RBV. American Association for the Study of Liver Diseases (AASLD) Liver Meeting; Boston, MA. 2014; abstract 1926

14. Nelson DR, Cooper JN, Lalezari JP, et al. All-oral 12-week treatment with daclatasvir plus sofosbuvir in patients with hepatitis C virus genotype 3 infection: ALLY-3 phase III study. Hepatology. 2015; 61:1127-1135. [PubMed: 25614962]

15. Wang C, Jia L, O'Boyle DR 2nd, et al. Comparison of daclatasvir resistance barriers on NS5A from hepatitis $\mathrm{C}$ virus genotypes 1 to 6 : implications for cross-genotype activity. Antimicrob Agents Chemother. 2014; 58:5155-5163. [PubMed: 24936600]

16. Tran, TT.; Morgan, TR.; Thuvulath, PJ. Safety and efficacy of treatment with sofosbuvir +GS-5816+/-ribavirin for 8 or 12 weeks in treatment naive patients with genotype 1-6 HCV infection. American Association for the Study of Liver Diseases (AASLD) Liver Meeting; November 7-12, 2014; Boston, MA. abstract 80

17. Zeuzem S, Ghalib R, Reddy KR, et al. Grazoprevir-elbasvir combination therapy for treatmentnaive cirrhotic and non-cirrhotic patients with chronic HCV genotype 1, 4, or 6 infection: a randomized trial. Ann Intern Med. 2015; 163:1-13. [PubMed: 25909356]

18. Chhatwal J, Kanwal F, Roberts MS, et al. Cost-effectiveness and budget impact of hepatitis C virus treatment with sofosbuvir and ledipasvir in the United States. Ann Intern Med. 2015; 162:397406. [PubMed: 25775312]

19. Kamal-Yanni M. Hepatitis C drug affordability. Lancet Glob Health. 2015; 3:e73-e74. [PubMed: 25617196]

20. Holmberg SD, Spradling PR, Moorman AC, et al. Hepatitis C in the United States. N Engl J Med. 2013; 368:1859-1861. [PubMed: 23675657]

21. Centers for Disease Control and Prevention. Recommendations for prevention and control of hepatitis C virus (HCV) infection and HCV-related chronic disease. MMWR Recomm Rep. 1998; 47(RR-19):1-39.

22. Smith BD, Morgan RL, Beckett GA, et al. Recommendations for the identification of chronic hepatitis C virus infection among persons born during 1945-1965. MMWR Recomm Rep. 2012; 61(RR-4):1-32. [PubMed: 22895429]

23. Ferrante JM, Winston DG, Chen PH, et al. Family physicians' knowledge and screening of chronic hepatitis and liver cancer. Fam Med. 2008; 40:345-351. [PubMed: 18465284]

24. Frazer K, Glacken M, Coughlan B, et al. Hepatitis C virus infection in primary care: survey of registered nurses' knowledge and access to information. J Adv Nurs. 2011; 67:327-339. [PubMed: 21044133]

25. Agrawal A, Mayo-Smith MF. Adherence to computerized clinical reminders in a large healthcare delivery network. Stud Health Technol Inform. 2004; 107(Pt 1):111-114. [PubMed: 15360785]

26. Armstrong GL, Wasley A, Simard EP, et al. The prevalence of hepatitis C virus infection in the United States, 1999 through 2002. Ann Intern Med. 2006; 144:705-714. [PubMed: 16702586]

27. Weinbaum C, Lyerla R, Margolis HS, et al. Prevention and control of infections with hepatitis viruses in correctional settings: Centers for Disease Control and Prevention. MMWR Recomm Rep. 2003; 52(RR-1):1-36. quiz CE1-CE4. [PubMed: 12562146] 
28. Galbraith JW, Franco RA, Donnelly JP, et al. Unrecognized chronic hepatitis C virus infection among baby boomers in the emergency department. Hepatology. 2015; 61:776-782. [PubMed: 25179527]

29. Delarocque-Astagneau E, Meffre C, Dubois F, et al. The impact of the prevention programme of hepatitis C over more than a decade: the French experience. J Viral Hepat. 2010; 17:435-443. [PubMed: 19780936]

30. de Scalioni LP, Cruz HM, de Paula VS, et al. Performance of rapid hepatitis C virus antibody assays among high- and low-risk populations. J Clin Virol. 2014; 60:200-205. [PubMed: 24794796]

31. McGowan CE, Fried MW. Barriers to hepatitis C treatment. Liver Int. 2012; 32(Suppl 1):151-156. [PubMed: 22212587]

32. Trooskin SB, Poceta J, Towey CM, et al. Results from a geographically focused, community-based HCV screening, linkage-to-care and patient navigation program. J Gen Intern Med. 2015; 30:950 957. [PubMed: 25680353]

33. Masson CL, Delucchi KL, McKnight C, et al. A randomized trial of a hepatitis care coordination model in methadone maintenance treatment. Am J Public Health. 2013; 103:e81-e88. [PubMed: 23947319]

34. Arora S, Thornton K, Murata G, et al. Outcomes of treatment for hepatitis C virus infection by primary care providers. N Engl J Med. 2011; 364:2199-2207. [PubMed: 21631316]

35. Knobler, S.; Lederberg, J.; Pray, LA., editors. Considerations for viral disease eradication: lessons learned and future strategies-workshop summary. National Academies Press; Washington, DC: 2002.

36. CDC. [Accessed April 10, 2014] Reported cases of acute, hepatitis C, by state: United States. 2007-2011. Available at: http://www.cdc.gov/hepatitis/Statistics/2011Surveillance/Table4.1.htm

37. Hepatitis C virus infection among adolescents and young adults: Massachusetts, 2002-2009. MMWR Morb Mortal Wkly Rep. 2011; 60:537-541. [PubMed: 21544042]

38. Notes from the field: hepatitis C virus infections among young adults—rural Wisconsin, 2010. MMWR Morb Mortal Wkly Rep. 2012; 61:358. [PubMed: 22592276]

39. Strickland GT. Liver disease in Egypt: hepatitis C superseded schistosomiasis as a result of iatrogenic and biological factors. Hepatology. 2006; 43:915-922. [PubMed: 16628669]

40. Hauri AM, Armstrong GL, Hutin YJ. The global burden of disease attributable to contaminated injections given in health care settings. Int J STD AIDS. 2004; 15:7-16. [PubMed: 14769164]

41. Apata IW, Averhoff F, Pitman J, et al. Progress toward prevention of transfusion-transmitted hepatitis B and hepatitis C infection: sub-Saharan Africa, 2000-2011. MMWR Morb Mortal Wkly Rep. 2014; 63:613-619. [PubMed: 25055184]

42. Mohamoud YA, Mumtaz GR, Riome S, et al. The epidemiology of hepatitis C virus in Egypt: a systematic review and data synthesis. BMC Infect Dis. 2013; 13:288. [PubMed: 23799878]

43. WHO. Guidelines for the screening, care and treatment of persons with hepatitis $\mathrm{C}$ infection. Geneva: WHO Press; 2014.

44. Chen TY, Ding EL, Seage GR III, et al. Meta-analysis: increased mortality associated with hepatitis C in HIV-infected persons is unrelated to HIV disease progression. Clin Infect Dis. 2009; 49:1605-1615. [PubMed: 19842982] 


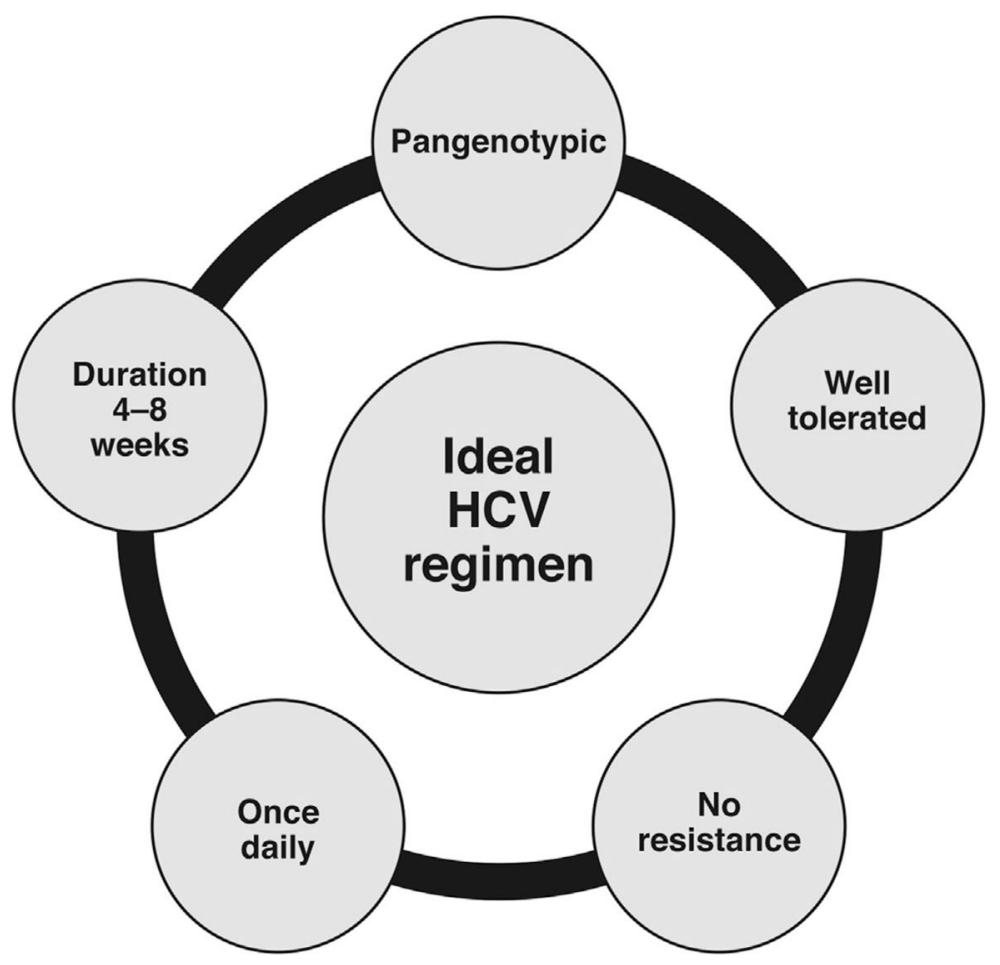

Figure 1.

Characteristics of a HCV regimen that would facilitate wide-scale care. 


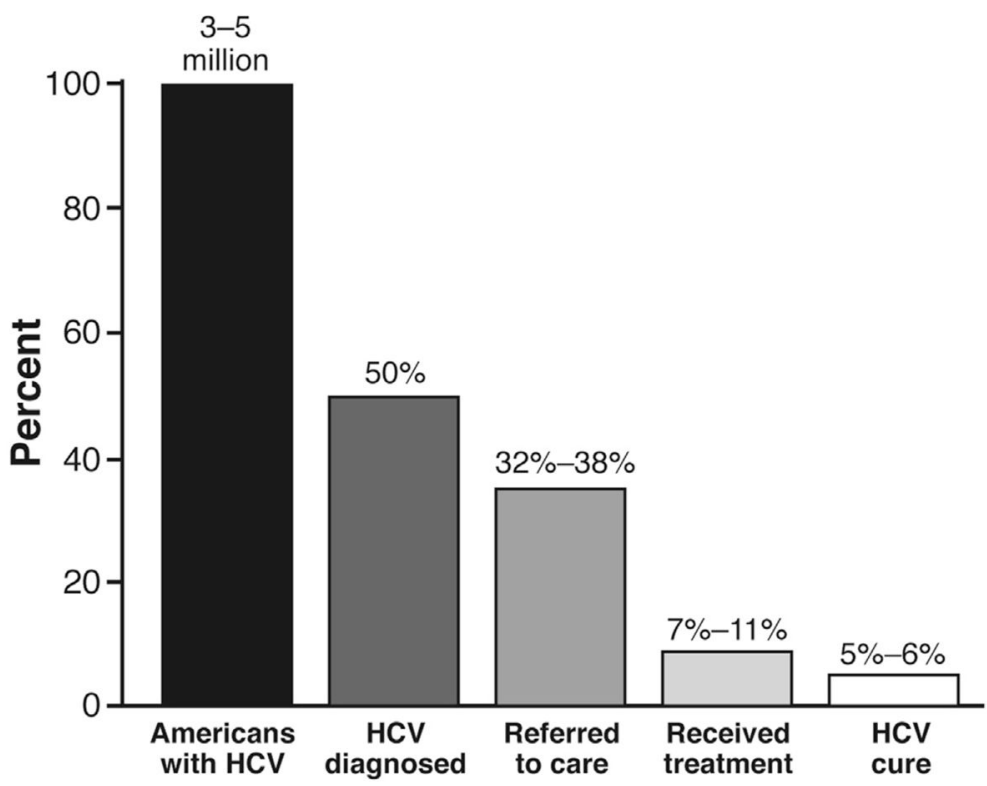

Figure 2.

Estimates of diagnosis and treatment rates of hepatitis $\mathrm{C}$ in the United States. 


\section{Table 1}

Populations With and Without Effective Interferon-free Regimens in 2015

\begin{tabular}{ll}
\hline Effective interferon-free regimens & Limitations with interferon-free regimens \\
\hline Genotypes 1, 2, 4 & Genotype 3, 5, 6 \\
HIV-HCV & Chronic kidney disease \\
Decompensated cirrhosis & Children \\
Post-liver transplant & \\
\hline
\end{tabular}

